3

4

5

\title{
Methodological considerations on selection of stable reference genes for RT- qPCR in the neonatal rat brain in hypoxia and hypothermia.
}

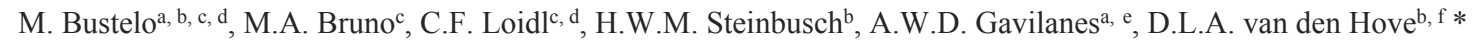

a. Department of Pediatrics, Maastricht University Medical Center (MUMC), Maastricht, the Netherlands

b. Department of Psychiatry and Neuropsychology, School for Mental Health and Neuroscience (MHeNs), Maastricht

University, Maastricht, the Netherlands

c. Instituto de Ciencias Biomédicas, Facultad de Ciencias Médicas, Universidad Católica de Cuyo, San Juan, Argentina

d. Laboratorio de Neuropatología Experimental, Instituto de Biología Celular y Neurociencia "Prof. E. De Robertis" (IBCN), Facultad de Medicina, Universidad de Buenos Aires, CONICET, Buenos Aires, Argentina

e. Instituto de Investigación e Innovación de Salud Integral, Facultad de Ciencias Médicas, Universidad Católica de Santiago de Guayaquil, Guayaquil, Ecuador

f. Department of Psychiatry, Psychosomatics and Psychotherapy, University of Würzburg, Würzburg, Germany

* Corresponding author

E-mail: d.vandenhove@maastrichtuniversity.nl (DVDH)

\section{Abstract}

Real-time reverse transcription PCR (qPCR) normalized to an internal reference gene (RG), is a frequently used method for quantifying gene expression changes in neuroscience. Although RG expression is assumed to be constantly independent of physiological or experimental conditions, several studies have shown that commonly used RGs are not expressed stably. The use of unstable RGs has a profound effect on the conclusions drawn from studies on gene expression, and almost universally results in spurious estimation of target gene expression. Approaches aimed at selecting and validating RGs often make use of different statistical methods, which may lead to conflicting results. The present study evaluates the expression of 5 candidate RGs (Actb, Pgkl, Sdha, Gapdh, Rnu6b) as a function of hypoxia exposure and hypothermic treatment in the neonatal rat cerebral cortex -in order to identify RGs that are stably expressed under these experimental conditions- and compares several statistical approaches that have been proposed to validate RGs. In doing so, we first analyzed the RG ranking stability proposed by several widely used statistical methods and related tools, i.e. the Coefficient of Variation (CV) analysis, GeNorm, NormFinder, 
31 BestKeeper, and the $\Delta \mathrm{Ct}$ method. Subsequently, we compared RG expression patterns between the various experimental groups. We found that these statistical methods, next to producing different rankings per se, all ranked RGs displaying significant differences in expression levels between groups as the most stable

34 RG. As a consequence, when assessing the impact of RG selection on target gene expression quantification, 35 substantial differences in target gene expression profiles were observed. As such, by assessing mRNA expression profiles within the neonatal rat brain cortex in hypoxia and hypothermia as a showcase, this study underlines the importance of further validating RGs for each new experimental paradigm considering the limitations of each selection method.

39 Keywords: Reference genes, QPCR, Neonatal Hypoxia-Ischemia.

\section{Abbreviations:}

42 qPCR real-time reverse transcription PCR

$43 \mathrm{RG}$ reference gene

44 Actb beta-actin

$45 \quad$ Pgk1 phosphoglycerate kinase 1

46 Sdha succinate dehydrogenase complex flavoprotein subunit A

47 Gapdh glyceraldehyde-3-phosphate dehydrogenase

$48 \quad$ Rnu6b U6 small nuclear RNA

49 18S rRNA 18 S ribosomal RNA

50 Hprt hypoxanthine-guanine phosphoribosyltransferase

$51 \quad B 2 m$ beta-2-micro-globulin

52 Tubb5 tubulin beta 5

53 Ppia peptidylprolyl isomerase A

54 Ywhaz tyrosine 3/tryptophan 5-monooxygenase activation protein zeta polypeptide

$55 \quad P g k 1 \quad$ phosphoglycerate kinase 1

$56 \quad$ Tbp TATAA-box binding protein

$57 \quad$ Arbp acidic ribosomal phosphoprotein P0

58 Gusb beta-glucuronidase

$59 \quad C k b \quad$ brain creatine kinase 
63 Rest repressor element 1-silencing transcription factor

64 Bad BCL2/BCL-XL-associated death promoter

\section{Introduction}

In qPCR analysis, reference genes (RGs) with stable expression levels are essential internal controls for relative quantification of mRNA expression. RGs normalize variations of candidate gene expression under different conditions $(1,2)$. The ideal RG should be expressed at constant levels regardless of e.g. experimental conditions, developmental stages or treatments $(3,4)$, and should have expression levels comparable to that of the target gene (5). Nevertheless, increasing evidence suggests that the expression of commonly used RGs often varies considerably under different experimental conditions, as reviewed previously $(6,7)$. The choice of unstable RGs for the normalization of qPCR data may give rise to inaccurate results, concomitant with potential expression changes in genes of interest being easily missed or overemphasized. Thus, the identification of stable RGs is a prerequisite for reliable qPCR experiments (9$11)$.

RG selection should be performed using the same samples that will be compared when looking at genes of interest. For this purpose, several statistical methods have been proposed, i.e. GeNorm (12), qBase (13), method (17). These statistical methods rank the stability of the candidate RGs based on a unique set of assumptions and associated algorithms. As a result, the predictions of these methods can differ significantly based on the method used, potentially leading to conflicting results. This observation has been frequently made, but still seems to be systematically ignored in recent validation studies.

To address this issue, several approaches that make use of several statistical approaches at the same time, have been proposed, including i) a weighted rank (18-20), an approach that is compromised by the fact that it does not consider the strengths and drawbacks of each method for a given experimental setting; ii) the 
"Geometric mean rank" that uses the average of the stability ranks across different methods yielding an overall ranking $(12,21)$; as well as iii) an integrated approach (22), including a first selection step making use of the $\mathrm{CV}$ analysis (eliminating genes with $\mathrm{CV}>50 \%$ ), and subsequently ranking the remaining genes using GeNorm.

In the present study, we compared these methods, on the evaluation of the stability of 5 candidate RGs in a murine model of perinatal asphyxia and therapeutic hypothermia. Perinatal asphyxia is a clinical condition defined as oxygen deprivation that occurs around the time of birth and may be caused by perinatal events such as placental abruption, cord-prolapse, or tight nuchal cord, limiting the supply of oxygenated blood to the fetus (23). Recently, hypothermia has emerged as the standard of care for perinatal asphyxia. Although this treatment has been demonstrated to be effective in reducing mortality and long-term consequences of perinatal asphyxia, the underlying mechanisms of this therapy are still not completely understood (24-28).

101 Assessing gene expression changes in the neonatal hypoxic-ischemic brain may be of added value in order

102 to further decipher the mechanism of perinatal asphyxia and to increase the effectivity of therapeutic 103 hypothermia and related therapies. Here, we used a murine perinatal hypoxic-ischemic encephalopathy 104 model (29-31) to address the abovementioned problems in RG selection and qPCR normalization. Several in vivo and in vitro studies on hypoxia, making use of qPCR, have been reported (19,32-39), indicating

106 that hypoxia significantly impacts the expression of various commonly used RGs. Although some of these 107 studies use the same or similar hypoxia models, the results vary substantially across studies, emphasizing 108 the need to publish these validation studies prior or parallel to reporting qPCR results.

110 We selected five candidate RGs based on published RG validation studies involving hypoxia (Table 1). 111 Subsequently, we applied various validated methodological and statistical methods to evaluate the effects

112 of anoxia and hypothermia on the expression stability of the candidate RGs. To evaluate the impact of the 113 resulting RG selection, we assessed the expression levels of the Repressor Element 1-Silencing 114 Transcription Factor (Rest), a gene that has been shown to be upregulated by hypoxic-ischemic injury in 115 the peri-infarct cortex of adult rats following transient focal ischemia induced by middle cerebral artery 116 occlusion (MCAO) (40). Moreover, the proapoptotic gene BCL2/BCL-XL-associated death promoter $117(\mathrm{Bad})$, a gene that has been shown to be up-regulated by hypoxia in the MCAO rat model, was assessed 

available under aCC-BY 4.0 International license.

118 (41). This study provides a basis for the selection of RGs and useful guidelines for future gene expression

119 studies, in particular regarding studies on developmental hypoxic insults.

120 Table 1. List of published RG validation studies involving hypoxia.

121 
bioRxiv preprint doi: https://doi.org/10.1101/793786; this version posted October 4, 2019. The copyright holder for this preprint (which was

not certified by peer review) is the author/funder, who has granted bioRxiv a license to display the preprint in perpetuity. It is made available under aCC-BY 4.0 International license.

122

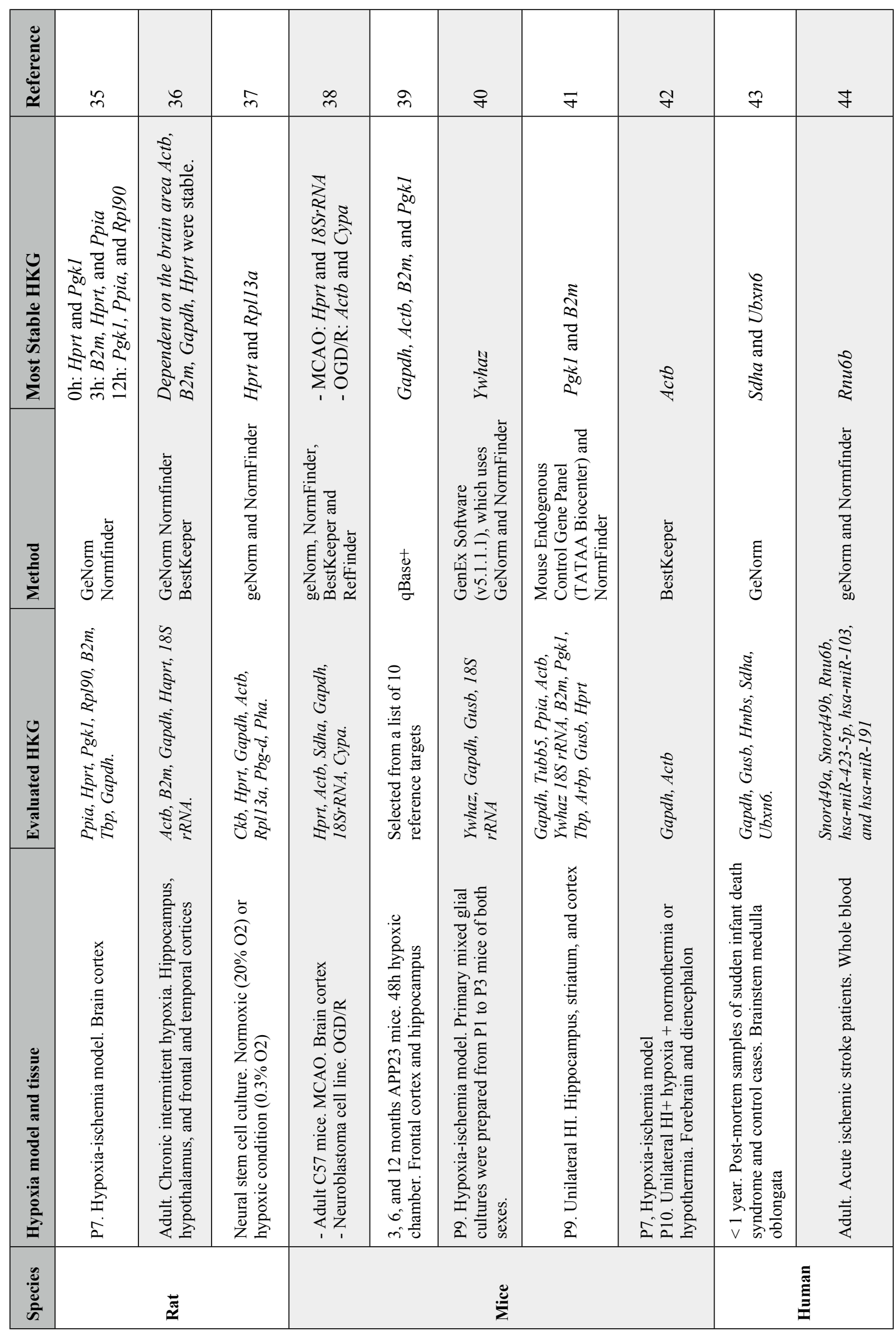




\section{Methods}

\section{Ethics statement}

126 This study was carried out in accordance with the recommendations in the Guide for the Care and Use of

127 Laboratory Animals of the National Institute of Health of Argentina. The protocol was approved by the

128 Biomedical Ethics Committee of Universidad Católica de Cuyo, San Juan, Argentina and by the Ethical

129 Committee of CICUAL: "Institutional Committee for the Use and Care of Laboratory Animals" (Resolution 130 no. 2079/07), Facultad de Medicina, Universidad de Buenos Aires, Argentina. Appropriate actions were 131 taken to minimize the number of animals used and their suffering, pain, and discomfort.

\section{Hypoxic-ischemic injury animal model}

134 Severe acute PA was induced using a model of hypoxia-ischemia as described previously (26-28). Briefly, 135 albino Sprague-Dawley rats were kept under standard laboratory conditions at $24^{\circ} \mathrm{C}$ with light-dark cycles 136 of 12:12 hours, and food and water present ad libitum. Fifteen timed-pregnant Sprague-Dawley rats were 137 used. The first group of offspring studied consisted of normally delivered naive pups that were used as 138 controls (CTL; $n=6)$. After vaginal delivery of the first pup, pregnant dams were euthanized by decapitation 139 and immediately hysterectomized. All full-term fetuses, still inside the uterus, were subjected to asphyxia

140 by transient immersion of both uterine horns in a saline bath for $20 \mathrm{~min}$ at either $37^{\circ} \mathrm{C}$ (perinatal asphyxia 141 in normothermia, PA, $\mathrm{n}=6$ ) or $15^{\circ} \mathrm{C}$ (perinatal asphyxia in hypothermia, [HYPPA]; $\mathrm{n}=6$ ). After asphyxia, 142 the uterine horns were opened, pups were removed, dried of delivery fluids, and stimulated to breathe, and 143 their umbilical cords were ligated. After recovery, one group of PA animals was placed on a cooling pad at

$1448^{\circ} \mathrm{C}$ for 15 minutes for hypothermic treatment (PAHYP, $\mathrm{n}=6$ ), while hypothermic control animals (HYP, $145 \mathrm{n}=6$ ) received the same treatment. After 15 minutes of exposure to the cold environment the core 146 temperature of the newborns was measured with a rectal probe (mean temperature: $20.1^{\circ} \mathrm{C} ; \mathrm{n}=8$ ). The pups 147 were subsequently placed under a heating lamp for recovery after which they were and placed with a 148 surrogate mother. Time of asphyxia was measured as the time elapsed from the hysterectomy up to the 149 recovery from the water bath. Pups that adjusted to the following parameters were included: 1. 150 Occipitocaudal length $>41 \mathrm{~mm}, 2$. Weight $>5 \mathrm{~g}$. 


\section{Total RNA extraction and reverse transcription cDNA}

\section{3 synthesis}

154 Animals were sacrificed by quick decapitation $24 \mathrm{~h}$ post treatment. The brain cortex was isolated, snap155 frozen in liquid nitrogen, ground into powder with pestle and mortar cooled in liquid nitrogen and then 156 stored at $-80^{\circ} \mathrm{C}$. Total RNA was isolated from about $80 \mathrm{mg}$ tissue powder using TRIzol ${ }^{(\text {Invitrogen Life }}$ 157 Technologies, USA) following the manufacturer's instructions. The residual DNA was removed by the 158 TURBO DNA free kit (Ambion Inc., UK). Yield and purity of RNA was determined by the NanoDrop ND1591000 spectrophotometer (Nanodrop technologies, USA). RNA samples with an absorbance ratio OD $160260 / 280$ between $1.9-2.2$ and OD 260/230 greater than 2.0 were used for further analysis. RNA integrity 161 was assessed using agarose gel electrophoresis. One microgram of RNA from each sample was reverse 162 transcribed using the High Capacity cDNA Reverse Transcription Kit (Applied Biosystems) according to 163 the manufacturer's instructions. cDNA was stored at $-20^{\circ} \mathrm{C}$ for future use. For qPCR analysis, each cDNA 164 sample was diluted 20 times with nuclease-free water.

\section{Real-time PCR}

167 Real-time PCRs were conducted using the LightCycler ${ }^{\circledR} 480$ Multiwell Plate 96 (Roche, Mannheim, 168 Germany) containing $1 \mu \mathrm{M}$ of each primer. For each reaction, the $20 \mu 1$ mixture contained $1 \mu 1$ of diluted 169 cDNA, 5 pmol each of the forward and reverse primers, and $10 \mu 12 \times$ SensiMix SYBR No-ROX Kit 170 (Bioline, UK). The amplification program was as follows: $95^{\circ} \mathrm{C}$ for $30 \mathrm{sec}, 40$ cycles at $95^{\circ} \mathrm{C}$ for $15 \mathrm{sec}$, 171 and $60^{\circ} \mathrm{C}$ for $15 \mathrm{sec}$, and $72^{\circ} \mathrm{C}$ for $15 \mathrm{sec}$. After amplification, a thermal denaturing cycle was conducted 172 to derive the dissociation curve of the PCR product to verify amplification specificity. Reactions for each 173 sample were carried out in triplicate. qPCR efficiencies in the exponential phase were calculated for each 174 primer pair using standard curves (5 ten-fold serial dilutions of pooled cDNA that included equal amounts 175 from the samples set). The mean threshold cycle (Ct) values for each serial dilution were plotted against 176 the logarithm of the cDNA dilution factor and calculated according to the equation $\mathrm{E}=10(-1 / \mathrm{slope}) \times 100$, 177 where the slope is the gradient of the linear regression line. 
179 Reference gene selection

180 Based on their common usage as endogenous control genes in previous studies (Table 1), five candidate

181 RGs were analyzed, i.e., Actb, Pgk1, Gapdh, Sdha, Rnu6b. These genes represent commonly used

182 endogenous control genes chosen from the relevant literature and have been previously validated in rat,

183 mouse and human brain tissues exposed to hypoxia. The selected RGs belong to different molecular

184 pathways to minimize the risk of co-regulation between genes. The primers were designed from nucleotide

185 sequences identified using NCBI BLAST (http://blast.ncbi.nlm.nih.gov/Blast.cgi). Rnu6b TaqMan

186 MicroRNA Assay $(R n u 6 b)$ was commercially available (Thermo Fisher Scientific, Product number:

187 4427975-001093). All other primers were ordered from Thermo Fisher Scientific with their certificates of

188 analysis. The primer characteristics of nominated RGs are listed in Table 2. The primer sequences $\left(5^{\prime}-3^{\prime}\right)$

189 of the target genes were as follows:

190 Rest; - F, AACTCACACAGGAGAACGCC - R, GAGGTTTAGGCCCGTTGTGA.

191 Bad; - F, GCCCTAGGCTTGAGGAAGTC - R, CAAACTCTGGGATCTGGAACA.

192

193 Table 2. List of RGs investigated by qPCR.

\begin{tabular}{|c|c|c|c|c|c|c|}
\hline $\begin{array}{l}\text { Gene } \\
\text { symbol }\end{array}$ & Gene name & $\begin{array}{l}\text { Accesion } \\
\text { number }\end{array}$ & Function & Primer sequence $\left(5^{\prime}-3^{\prime}\right)$ & $\begin{array}{l}\text { Product } \\
\text { length } \\
\text { (bp) }\end{array}$ & $\begin{array}{c}\text { Efficiency } \\
(\%)\end{array}$ \\
\hline$A c t b$ & Beta-actin & NM_031144 & Cytoskeletal structural protein & $\begin{array}{l}\text { F: CCCGCGAGTACAACCTTCTTG } \\
\text { R: GTCATCCATGGCGAACTGGTG }\end{array}$ & 71 & 104.3 \\
\hline Pgkl & $\begin{array}{l}\text { Phosphoglycerate } \\
\text { kinase } 1\end{array}$ & NM_053291.3 & Glycolytic enzyme & $\begin{array}{l}\text { F: GTCGTGATGAGGGTGGACTT } \\
\text { R: AACCGACTTGGCTCCATTGT }\end{array}$ & 120 & 99.75 \\
\hline Sdha & $\begin{array}{c}\text { Succinate } \\
\text { dehydrogenase } \\
\text { complex flavoprotein } \\
\text { subunit A }\end{array}$ & NM_130428.1 & $\begin{array}{l}\text { Catalytic subunit of succinate- } \\
\text { ubiquinone oxidoreductase }\end{array}$ & $\begin{array}{l}\text { F: AGCCTCAAGTTCGGGAAAGG } \\
\text { R: CCGCAGAGATCGTCCATACA }\end{array}$ & 102 & 102.75 \\
\hline Gapdh & $\begin{array}{l}\text { Glyceraldehyde-3- } \\
\text { phosphate } \\
\text { dehydrogenase }\end{array}$ & NM_017008.4 & $\begin{array}{l}\text { Membrane fusion, microtubule } \\
\text { bundling, cell death, and neurite } \\
\text { outgrowth }\end{array}$ & $\begin{array}{l}\text { F: AAGGGCTCATGACCACAGTC } \\
\text { R: GTGAGCTTCCCATTCAGCTC }\end{array}$ & 143 & 92.1 \\
\hline Rnu6b & $\begin{array}{l}\text { RNU6-2; U6 small } \\
\text { nuclear RNA }\end{array}$ & NR_002752 & ncRNAs & $\begin{array}{l}\text { CGCAAGGATGACACGCAAATTCG } \\
\text { TGAAGCGTTCCATATTTTT }\end{array}$ & 64 & 93.95 \\
\hline
\end{tabular}

194

195 Analysis of expression stability using multiple statistical approaches. To assess the stability of

196 candidate RGs, five statistical methods, each with unique characteristics, were used: GeNorm, BestKeeper,

197 NormFinder, Coefficient of Variation analysis, and the comparative $\Delta \mathrm{Ct}$ method. Ct values were converted

198 to non-normalized relative quantities according to the formula: 2- $\triangle \mathrm{Ct}$. $\mathrm{CV}$ analysis, GeNorm and 
199 NormFinder calculations are based on these converted quantities, whereas BestKeeper and the $\Delta \mathrm{Ct}$ method

200 make use of raw Cq values.

201

202 Impact of selection of RGs on gene expression normalization. The impact of RG selection on gene expression quantification was assessed via examining the expression of Rest and Bad. Six gene expression normalizing strategies were used to represent the least and most stable reference genes. The relative expression profiles of Rest and Bad were determined and normalized with all tested RGs. Relative fold changes in gene expression were calculated using the DDCt and Pfaffl methods. Data was expressed as mean \pm standard error of the mean (SEM) from six independent samples/group with triple qPCR reactions. One-way analysis of variance (ANOVA) test was applied to analyze significant differences between conditions for each house-keeping gene. Differences were reported as statistically significant when $p<0.05$. GraphPad Prism 6 (GraphPad Software, USA) was used for statistical procedures and graph plotting.

\section{Results}

\section{3 qPCR}

214 Pilot assays were performed to optimize cDNA and primer quantities. A total of 0.9 mg of RNA that was

215 previously treated with DNase was used for the reverse transcription reaction in a total volume of $40 \mathrm{ml}$.

216 One microliter of the resulting cDNA was used for the qPCR reaction. Each gene amplification was

217 analyzed, and a melting curve analysis was performed, showing a single peak indicating the temperature of

218 dissociation. Efficiencies are shown in Table 2. All Ct values were between 17.0 and 33.0.

\section{Coefficient of Variation analysis}

220 We calculated the raw expression profiles of RGs as changes of $\mathrm{Ct}$ values across groups and ranked the 221 gene stability according to the CV. The CV analysis is a descriptive statistical method where the Ct values 222 of all candidate RGs across samples are first linearized (2-Cq). Next, the CV for each gene across all samples 223 was calculated and expressed as a percentage. The CV estimates the variation of a gene across all samples 224 taken together, and, therefore, a lower CV value indicates higher stability. This analysis on the cortical samples revealed Gapdh as the most stable RG, and Actb as the least stable RG. This method however does 
226 not consider the variation across different treated groups; hence, CV analysis alone cannot determine the best set of RGs. caps denote the maximum and minimum values. b. CV analysis of the linearized $\mathrm{Ct}$ values.

234 To assess if the mean mRNA levels across groups were significantly different from one another, a Oneway ANOVA was used. The results demonstrated that variations in the Ct values for the different treatments were different for all candidate RGs. Four of the five genes tested (Sdha, Rnu6b, Pgk1, Actb) showed significant variation in mRNA levels across different treatments (Fig 2). Only Gapdh showed no significant changes. These results, making use of the raw expression profiles of the RGs, suggest that the various experimental conditions were associated with changes in RG expression levels that, as such, could skew

240 the normalized profile of target genes. As a result, RG selection without accounting for potential expression

241 differences between conditions is accompanied by a significant bias in the results and their interpretation.

242 Hence, it is of utmost importance to validate the stability of RGs prior to normalization in gene expression studies.

Fig 2. Expression profiles of RG expressed as Cp across the experimental conditions. a. Actb, b. Pgk1,

246 c. Sdha. d. Gapdh, e. Rnu6b. Results are expressed as the Mean \pm SEM for each treatment. One-way

247 ANOVA was performed to asses differences between the means of all groups. Statistical significance is denoted by $p$ values: $* p<0.05, * * p<0.01, * * * p<0.001$. known statistical methods (Table 3). 
Table 3. Candidate RG expression stability.

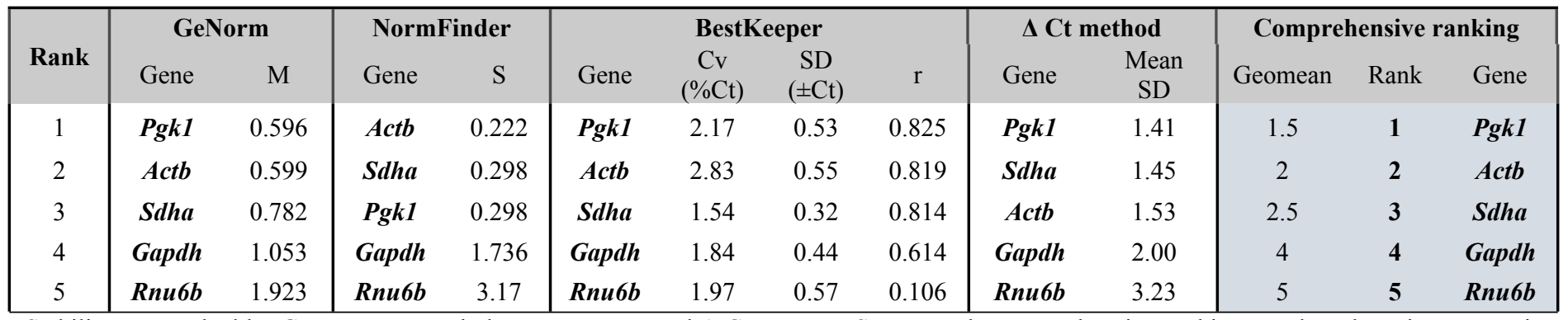

Stability was ranked by GeNorm, NormFinder, BestKeeper and $\Delta$ CT average STDEV. The comprehensive ranking was based on the geometric mean of the gene rank. Candidates are listed from top to bottom in order of decreasing expression stability. (SD [ $\pm \mathrm{Ct}$ ]: standard deviation of the $\mathrm{Ct}$; $\mathrm{CV}[\% \mathrm{Ct}]$ : coefficient of variance expressed as a percentage of the $\mathrm{Ct}$ level; geomean: geometrical mean).

258 First, a GeNorm analysis was performed on all five candidate genes. GeNorm calculates stability value (M)

259 based on pairwise variation of every two genes. The rationale is that if two genes vary similarly across all

260 samples, then they are the most stable RGs for that dataset. A limitation of this method is that if two genes

261 are regulated in the same direction by one or more experimental conditions, those will often be assumed to

262 be the most stable. In our analysis, except for Rnu6b, which presented the highest M-value (M=1.923), all

263 of the other candidate RGs presented M-values lower than 1.5, which is considered to be the cut-off for

264 suitability. Based on this analysis for the neonatal cortex, the most stable RGs were Pgkl and Actb. This is

265 in stark contrast to the CV analysis, that showed those genes as the least stable ones (higher CV), and to

266 the expression profiles that showed inter-group differences.

268 NormFinder calculates the stability score (S) based on the inter- and intra-group variation. However, it has

269 been reported that including genes with high overall variation can affect the stability ranking of all genes

270 with this method (22). This algorithm can potentially be improved after identifying and removing genes

271 with high overall variation.. Actb, Sdha and $P g k 1$ were the most stable RGs, presented stability values lower

272 than 0.3. Gapdh $(\mathrm{SV}=1.736)$ and Rnu6b $(\mathrm{SV}=3.17)$ were the least stable.

273

274 BestKeeper uses the cycle threshold (Ct) values to calculate a standard deviation (SD), coefficient of

275 variance (CV), and Pearson correlation coefficient (r) for each gene. Lower SD and CV values indicate

276 more stable gene expression, and genes that exhibit a SD in $\mathrm{Ct}$ values above 1.0 should be eliminated and

277 regarded as unreliable controls. Then, the remaining RG are ranked according to $r$ values, with a higher $r$

278 value indicating more stable gene expression. None of the genes analyzed were excluded for having SD 
above 1. The most stable RG was Pgk1 (r=0.825), while Rnu6b was considered the least stable gene $(\mathrm{r}=0.106)$. The ranking obtained from this analysis was the same as the one obtained with GeNorm.

281

282 The $\Delta$-Ct method works on the same rationale as GeNorm but calculates the stability value (mean SD) differently; it is calculated as the average standard deviation of the Ct value differences that the gene exhibits with other genes. Using this method, the ranking was similar to previous rankings. The most stable RGs were Pgkl (Av. $\mathrm{SD}=1.41$ ) and $S d h a(\mathrm{Av} \cdot \mathrm{SD}=1.45)$, and the least stable Rnu6b (Av.SD=3.23). The overall ranking depicted in Table 3 was based on the geometric mean of the previous gene ranks. This ranking indicates that for this tissue and treatment, the most stable RG was Pgkl.

\section{Impact of RG selection on target gene expression profiles}

The impact of RG selection on gene expression quantification was assessed by examining the expression of Rest and Bad. These genes have shown to be influenced by hypoxia and hypothermia. Five gene expression normalizing strategies were used to select the least and most stable RGs, and the best combination of two genes, Actb/Pgkl (Fig 3). Expression values were calculated relative to expression in control animals, using both the $\Delta \Delta$ Ct method (Livak \& Schmittgen, 2001) and the primer efficiency method (Pfaffl, 2001, Fig 3). Results were similar using Livak or Pfaffl methods. As expected, even when the general pattern of target gene expression was similar for most of the RGs across treatments, target gene expression levels were different depending on the RG used for normalization causing differences in the significance level of the expression patterns.

Fig 3. Evaluation of the impact of selection of RG on gene expression normalization. Expression profiles of Rest and Bad normalized by different strategies. Arithmetic mean values and standard deviations were obtained from three bioreplicates.

\section{Discussion}

305 The selection of RGs in qPCR experiments has an enormous impact on the reliability and interpretation of 
307 that normalization of qPCR results against a single RG is likely to be inadequate and that normalization

308 against a panel of RGs containing at least three stable RGs is preferred. However, for most of the RGs used

309 in published qPCR studies, no thorough investigation of their variation over experimental conditions has

310 been performed and/or reported (48). Many researchers continue to use a single, unvalidated RG to

311 normalize data.

312

313 The majority of studies where assessment of the RGs' stability is included make use of statistical tools like

314 GeNorm, BestKeeper, NormFinder, CV analysis, and the comparative $\Delta \mathrm{Ct}$ method. Each of these methods

315 determines the stability based on a set of assumptions and calculations, and has its own limitations. In

316 general, methods that rely on pairwise variation (GeNorm and Pairwise $\Delta \mathrm{Ct}$ method) are influenced by the

317 expression pattern of all genes making their ranking inter-dependent. The CV analysis does not take the

318 variation between groups into account, hence alone it cannot determine the best (set of) RG(s), but it can

319 be used as a first filter to discard genes with high overall variance. Moreover, except for the CV analysis,

320 the presence of genes with high overall variation impact upon the ranking of all these methods.

321

322 As a result, the selection of stable RGs varies significantly depending on the method used making the choice

323 of the validation method a critical step in qPCR assays. In our study, using Geomean, Pgkl was the most

324 stable gene across treatments, while U6 and Gapdh were ranked as most variable. This is in stark contrast

325 to the CV\% Analysis and intergroup ANOVA Ct variations that indicated that Gapdh was the most stable

326 gene among groups, and Actb the least stable.

327

328 Using any of these methods alone is not sufficient in obtaining bias-free results. Generally, stability

329 validation studies have ranked the genes using Geomean, a ranking obtained from the mean rank of the

330 statistical tools used. This method does not take into account the limitations of each algorithm separately,

331 which is why it is increasingly considered an erroneous approach when validating RGs. This makes the

332 identification of the best RGs very unwieldy. Using the same statistical methods, new approaches have

333 been proposed, such as the "Integrated approach" (22) that has shown to provide a more accurate estimate

334 of RG stability. It is advisable to devise integrated approaches based on suitability for each experimental

335 setting. 

with the different methodologies, and the associated bias was reflected in our target gene quantification.

339 Our study emphasizes the necessity of validating RGs previous to assessing target gene qPCR data, and the importance of choosing the right set of statistical methods for doing so. Such an approach would lead to more accurate and reproducible expression assessment.

\section{Funding}

344 This research was partially supported by the Sistema de Investigación y Desarrollo (SINDE) and the

345 Vicerrectorado de Investigación y Posgrado of the Universidad Católica de Santiago de Guayaquil,

346 Guayaquil, Ecuador. M. Bustelo is funded by Consejo Nacional de Investigaciones Científicas y Técnicas

347 (CONICET) of Argentina and the Foundation of Pediatrics, Maastricht University Medical Center. F. Loidl

348 is supported by Universidad de Buenos Aires (UBACyT - 20020160100150BA).

\section{References}

351 1. Schmittgen TD, Livak KJ. Analyzing real-time PCR data by the comparative C(T) method. Nat Protoc. 2008;

2. Ruijter JM, Ramakers C, Hoogaars WMH, Karlen Y, Bakker O, van den hoff MJB, et al. Amplification efficiency: Linking baseline and bias in the analysis of quantitative PCR data. Nucleic Acids Res. 2009;

356 3. Wong ML, Medrano JF. Real-time PCR for mRNA quantitation. Biotechniques. 2005;

357 4. Bustin SA, Benes V, Garson JA, Hellemans J, Huggett J, Kubista M, et al. The MIQE guidelines: Minimum information for publication of quantitative real-time PCR experiments. Clin Chem. 2009;

5. Suzuki T, Higgins PJ, Crawford DR. Control selection for RNA quantitation. BioTechniques. 2000.

360 6. Bustin SA, Wittwer CT. MIQE: A Step Toward More Robust and Reproducible Quantitative PCR. Clin Chem. 2017;

362 7. Bustin SA, Benes V, Garson J, Hellemans J, Huggett J, Kubista M, et al. The need for transparency and good practices in the qPCR literature. Nature Methods. 2013.

364 8. Coulson DTR, Brockbank S, Quinn JG, Murphy S, Ravid R, Brent GB, et al. Identification of valid reference genes for the normalization of RT qPCR gene expression data in human brain tissue. 
368 10. Tunbridge EM, Eastwood SL, Harrison PJ. Changed relative to what? Housekeeping genes and normalization strategies in human brain gene expression studies. Biological Psychiatry. 2011.

11. Huggett JF, Foy CA, Benes V, Emslie K, Garson JA, Haynes R, et al. The digital MIQE guidelines: Minimum information for publication of quantitative digital PCR experiments. Clin Chem. 2013; data by geometric averaging of multiple internal control genes. Genome Biol. 2002;

13. Hellemans J, Mortier G, De Paepe A, Speleman F, Vandesompele J. qBase relative quantification framework and software for management and automated analysis of real-time quantitative PCR data. Genome Biol. 2008;

14. Pfaffl MW, Tichopad A, Prgomet C, Neuvians TP. Determination of stable housekeeping genes, differentially regulated target genes and sample integrity: BestKeeper - Excel-based tool using pairwise correlations. Biotechnol Lett. 2004;

15. Andersen CL, Jensen JL, Ørntoft TF. Normalization of real-time quantitative reverse transcriptionPCR data: A model-based variance estimation approach to identify genes suited for normalization, applied to bladder and colon cancer data sets. Cancer Res. 2004;

16. Boda E, Pini A, Hoxha E, Parolisi R, Tempia F. Selection of reference genes for quantitative realtime RT-PCR studies in mouse brain. J Mol Neurosci. 2009;

17. Silver N, Best S, Jiang J, Thein SL. Selection of housekeeping genes for gene expression studies

18. Perez LJ, Rios L, Trivedi P, D'Souza K, Cowie A, Nzirorera C, et al. Validation of optimal reference genes for quantitative real time PCR in muscle and adipose tissue for obesity and diabetes research. Sci Rep. 2017;

19. Kang Y, Wu Z, Cai D, Lu B. Evaluation of reference genes for gene expression studies in mouse and N2a cell ischemic stroke models using quantitative real-time PCR. BMC Neurosci. 2018; reference genes for normalization in quantitative RT-PCR. BMC Bioinformatics. 2010; 
396 22. Sundaram VK, Sampathkumar NK, Massaad C, Grenier J. Optimal use of statistical methods to validate reference gene stability in longitudinal studies Abstract : 2019;

398 23. Fattuoni C, Palmas F, Noto A, Fanos V, Barberini L. Perinatal asphyxia: A review from a metabolomics perspective. Molecules. 2015;

24. Dixon K, Smith S. In neonates with hypoxic ischemic encephalopathy, is therapeutic hypothermia outside of current criteria safe? A literature review. Journal of Neonatal Nursing. 2019.

25. Davidson JO, Wassink G, van den Heuij LG, Bennet L, Gunn AJ. Therapeutic hypothermia for neonatal hypoxic-ischemic encephalopathy - Where to from here? Frontiers in Neurology. 2015.

26. Shankaran S, Pappas A, McDonald SA, Vohr BR, Hintz SR, Yolton K, et al. Childhood outcomes after hypothermia for neonatal encephalopathy. Obstetrical and Gynecological Survey. 2012.

27. Gluckman PD, Wyatt JS, Azzopardi D, Ballard R, Edwards AD, Ferriero DM, et al. Selective head cooling with mild systemic hypothermia after neonatal encephalopathy: Multicentre randomised trial. Lancet. 2005;

28. Iwata O, Iwata S, Thornton JS, De Vita E, Bainbridge A, Herbert L, et al. "Therapeutic time hypothermia and gender on survival and behavior after perinatal asphyxia in rats. Physiol Behav.

30. Capani F, Loidl CF, Aguirre F, Piehl L, Facorro G, Hager A, et al. Changes in reactive oxygen species (ROS) production in rat brain during global perinatal asphyxia: An ESR study. Brain Res. 2001;

31. Loidl CF, Capani F, López-Costa JJ, Selvín-Testa A, López EM, Pecci-Saavedra J. Long term changes in NADPH-diaphorase reactivity in striatal and cortical neurons following experimental perinatal asphyxia: Neuroprotective effects of hypothermia. Int J Neurosci. 1997; 
426 34. Yao L, Chen X, Tian Y, Lu H, Zhang P, Shi Q, et al. Selection of housekeeping genes for normalization of RT-PCR in hypoxic neural stem cells of rat in vitro. Mol Biol Rep. 2012;

428 35. A.A. B, Y. M, R. V, V.G. S, P. S, M. R, et al. Does Caspase-6 Have a Role in Perinatal Brain Injury? Dev Neurosci. 2015;

430 36. Järlestedt K, Rousset CI, Faiz M, Wilhelmsson U, Stahlberg A, Sourkova H, et al. Attenuation of reactive gliosis does not affect infarct volume in neonatal hypoxic-ischemic brain injury in mice. PLoS One. 2010;

37. Keddy PGW, Dunlop K, Warford J, Samson ML, Jones QRD, Rupasinghe HPV, et al. Neuroprotective and Anti-Inflammatory Effects of the Flavonoid-Enriched Fraction AF4 in a Mouse Model of Hypoxic-Ischemic Brain Injury. PLoS One. 2012;

38. El-Kashef N, Gomes I, Mercer-Chalmers-Bender K, Schneider PM, Rothschild MA, Juebner M. Validation of adequate endogenous reference genes for reverse transcription-qPCR studies in MicroRNA-210 Improves Long-Term Outcomes after Focal Cerebral Ischemia in Mice. CNS Neurosci Ther. 2016;

442 40. Morris-Blanco KC, Kim TH, Bertogliat MJ, Mehta SL, Chokkalla AK, Vemuganti R. Inhibition of the Epigenetic Regulator REST Ameliorates Ischemic Brain Injury. Molecular Neurobiology. 2018 ; 
a. b.

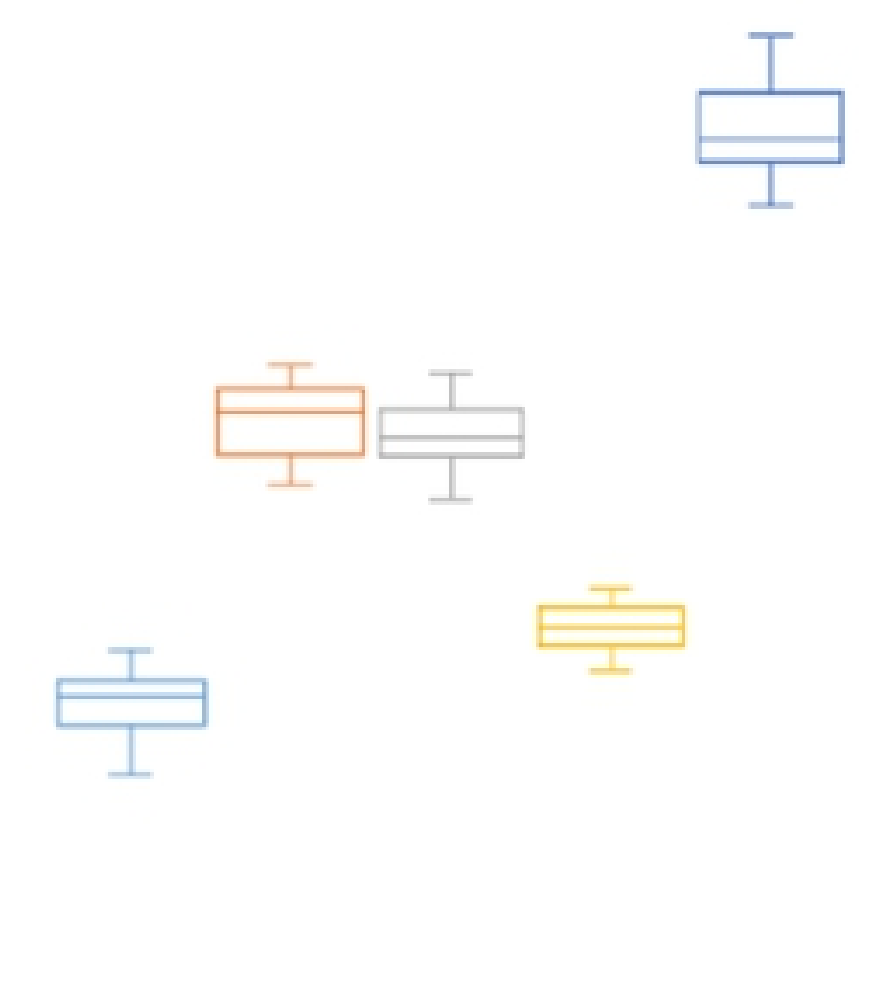

$\square$ Actb $\square$ Pgk1 $\square$ Sdha $\square$ Gapdh $\square$ RnU6b

Figure 1

\begin{tabular}{|c|c|c|}
\hline \multicolumn{3}{|c|}{ CV Analysis } \\
\hline Gene & CV\% & Rank \\
\hline Gapdh & 27.89 & 1 \\
\hline Sdha & 39.67 & 2 \\
\hline Pgk1 & 44.51 & 3 \\
\hline Rnu6b & 46.34 & 4 \\
\hline Actb & 47.42 & 5 \\
\hline
\end{tabular}



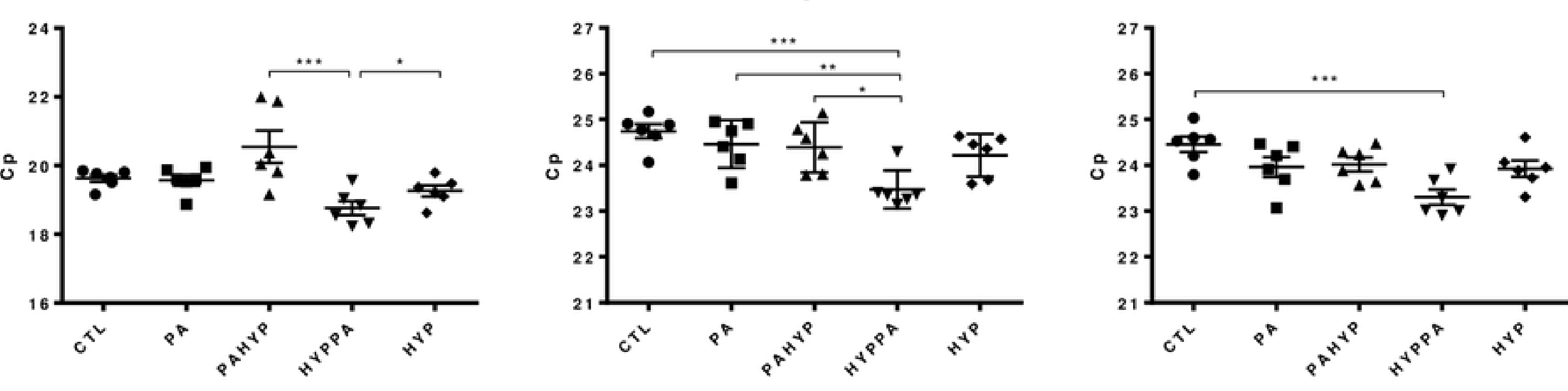

G A PDH
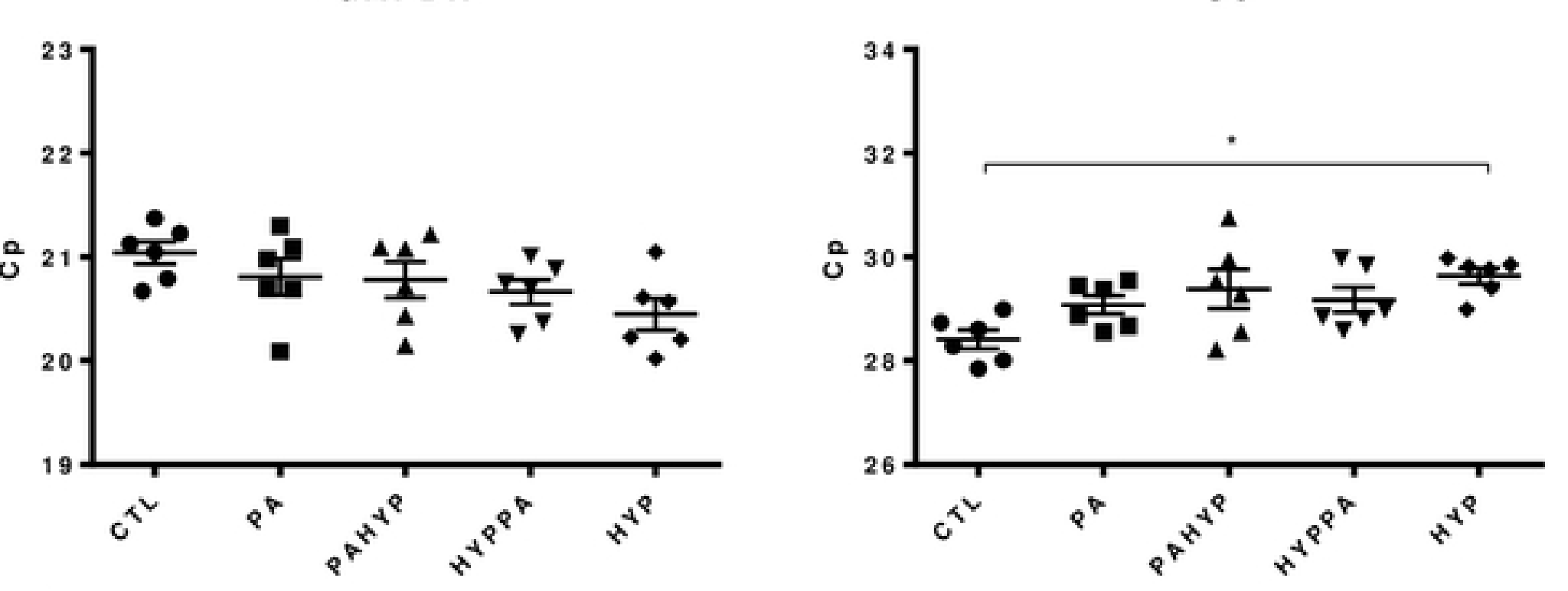

Figure 2 

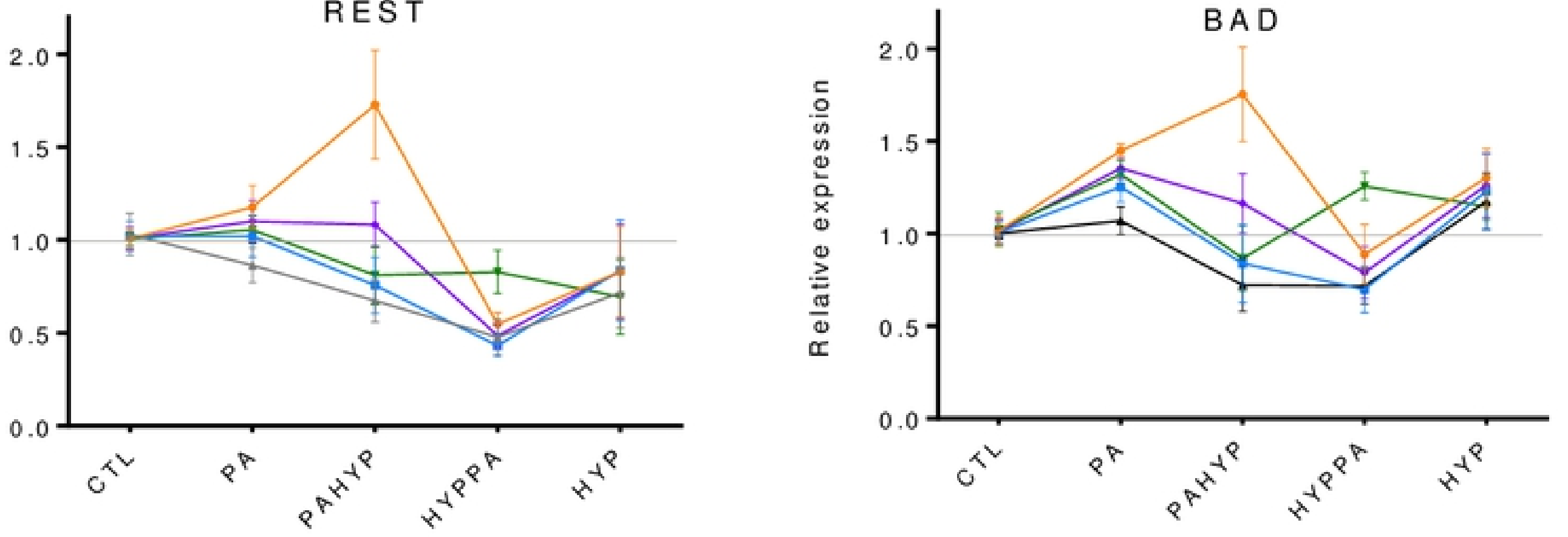

$=$ Pgk1

$\because A c t b$

$\rightarrow$ Actb, Pgk 1

$\rightarrow$ Sdha

$\rightarrow$ Gapdh

Figure 3 